\title{
The local wave phenomenon in the quintic nonlinear Schrödinger equation by numerical methods
}

\author{
Yaning Tang ( $\nabla$ tyaning@nwpu.edu.cn ) \\ Northwestern Polytechnical University \\ Zaijun Liang \\ Northwestern Polytechnical University \\ Wenxian Xie \\ Northwestern Polytechnical University
}

\section{Research Article}

Keywords: Quintic nonlinear Schrödinger equation, Spectral signatures, Symmetrical split-step Fourier method, Modulation instability, Rogue waves

Posted Date: August 30th, 2021

DOl: https://doi.org/10.21203/rs.3.rs-835181/v1

License: (9) This work is licensed under a Creative Commons Attribution 4.0 International License. Read Full License

Version of Record: A version of this preprint was published at Nonlinear Dynamics on February 2nd, 2022. See the published version at https://doi.org/10.1007/s11071-021-07169-6. 


\title{
The local wave phenomenon in the quintic nonlinear Schrödinger equation by numerical methods
}

\author{
Yaning Tang*, Zaijun Liang, Wenxian Xie \\ School of Mathematics and Statistics, Northwestern Polytechnical University, \\ Xi'an, Shaanxi 710129, P.R. China
}

\begin{abstract}
The nonlinear Schrödinger hierarchy has a wide range of applications in modeling the propagation of light pulses in optical fibers. In this paper, we focus on the integrable nonlinear Schrödinger (NLS) equation with quintic terms, which play a prominent role when the pulse duration is very short. First, we investigate the spectral signatures of the spatial Lax pair with distinct analytical solutions and their periodized wavetrains by Fourier oscillatory method. Then, we numerically simulate the wave evolution of the quintic NLS equation from different initial conditions through the symmetrical split-step Fourier method. We find many localized high-peak structures whose profiles are very similar to the analytical solutions, and we analyze the formation of rouge waves (RWs) in different cases. These results may be helpful to understand the excitation of nonlinear waves in some nonlinear fields, such as optical fibers, oceanography and so on.
\end{abstract}

Keywords:

Quintic nonlinear Schrödinger equation; Spectral signatures; Symmetrical split-step Fourier method; Modulation instability; Rogue waves

\section{Introduction}

The analytical solutions of the nonlinear evolution equations are widely applied in many fields, such as nonlinear optical fiber, plasma physics and so on [1,2]. In the past decades, scholars have carried out a lot of research on them [3-10]. In particular, as a type of extreme nonlinear waves, rouge waves (RWs) with high amplitudes and huge energies have attracted extensive attention in oceanography, nonlinear optics, Bose-Einstein condensates, plasma physics, and even financial system [11-16].

Some previous studies have investigated the physical mechanisms of RWs in the focusing NLS equation from the different viewpoints [17-20]. Particularly, modulation instability (MI) plays an important role in the generation of RWs [21, 22]. Recently, the formation of RWs in the focusing NLS equation and Hirota equation has been studied by inverse scattering analysis method from the viewpoint of their spectral problems [19, 23].

In this paper, we numerically analyze spectral signatures and the formation of RWs in the

\footnotetext{
${ }^{*}$ Corresponding author.

Email address: tyaning@nwpu.edu.cn (Yaning Tang)
} 
integrable quintic NLS equation [24],

$$
i u_{t}+\frac{1}{2}\left(u_{x x}+2|u|^{2} u\right)-i \delta\left(u_{x x x x x}+10|u|^{2} u_{x x x}+10\left(\left|u_{x}\right|^{2} u\right)_{x}+20 u^{*} u_{x} u_{x x}+30|u|^{4} u_{x}\right)=0
$$

by using the numerical inverse scattering transformation (IST) method. Here $t$ is the propagation variable, $x$ is the transverse variable and the modulus of complex valued function $u(x, t)$ represents the envelope of the nonlinear wave. The real constant $\delta$ stands for the fifth-order dispersion. Eq. (1) can be used to model the propagation of light pulses in optical fibers and the quintic terms play a prominent role when the pulse duration is very short. It is a compatibility condition, $\Phi_{x t}=\Phi_{t x}$, of the system of two linear partial differential equations with variable coefficients (also known as the Lax pair) [25],

$$
\begin{gathered}
\Phi_{x}=X \Phi, \quad X(x, t, \lambda)=i \lambda \sigma+Q, \\
\Phi_{t}=T \Phi, \quad T(x, t, \lambda)=\sum_{j=0}^{5} \lambda^{j} T_{j}
\end{gathered}
$$

where $\lambda$ is a complex spectral parameter, $\Phi$ is a column vector given by

$$
\Phi=\Phi(x, t)=\left(\begin{array}{c}
\Phi_{1}(x, t) \\
\Phi_{2}(x, t)
\end{array}\right)
$$

with $\Phi_{1}(x, t)$ and $\Phi_{2}(x, t)$ being complex valued functions, and matrices $\sigma, Q, T_{j}(j=0, \cdots, 5)$ are given by

$$
\begin{aligned}
& \sigma=\left(\begin{array}{cc}
1 & 0 \\
0 & -1
\end{array}\right), Q=\left(\begin{array}{cc}
0 & u^{*} \\
u & 0
\end{array}\right), T_{j}=i\left(\begin{array}{cc}
A_{j} & B_{j}^{*} \\
B_{j} & -A_{j}
\end{array}\right), \\
& A_{5}=16 \delta, B_{5}=0, A_{4}=0, B_{4}=16 \delta u, A_{3}=-8 \delta|u|^{2}, B_{3}=8 i \delta u_{x}, \\
& A_{2}=1+4 i \delta\left(u_{x}^{*} u-u_{x} u^{*}\right), \quad B_{2}=-8 \delta|u|^{2} u-4 \delta u_{x x}, \\
& A_{1}=C_{1}+6 \delta|u|^{4}+2 \delta\left(u_{x x}^{*} u-\left|u_{x}\right|^{2}+u_{x x} u^{*}\right), \quad B_{1}=u-12 i \delta|u|^{2} u_{x}-2 i \delta u_{x x x}, \\
& A_{0}=\frac{1}{2} C_{0}-\frac{1}{2}|u|^{2}-i \delta\left(u_{x x x}^{*} u-u_{x x}^{*} u_{x}+u_{x x} u_{x}^{*}-u_{x x x} u^{*}\right)-6 i \delta\left(u_{x}^{*} u-u_{x} u^{*}\right)|u|^{2}, \\
& B_{0}=C_{1} u+6 \delta|u|^{4} u+\frac{1}{2} i u_{x}+2 \delta u_{x x}^{*} u^{2}+4 \delta\left|u_{x}\right|^{2} u+6 \delta\left(u_{x}\right)^{2} u^{*}+8 \delta u_{x x}|u|^{2}+\delta u_{x x x x},
\end{aligned}
$$

where $C_{0}$ and $C_{1}$ are arbitrary real constants, and the superscript "*" represents the complex conjugate.

The rest of this paper is arranged as follows. In section 2, we numerically study the spectral signatures of the spatial Lax pair (2a) with distinct potentials of the quintic NLS equation through the Fourier collocation method. In section 3, we discuss the formation of rogue waves in the quintic NLS equation. Distinct non-zero initial conditions are used to analyze the generation mechanism of rogue waves. In section 4, we summarize the paper. 


\section{Analytical solutions and their spectral portraits}

\subsection{Fundamental analytical solutions}

According to the previous researches, Eq. (1) has been proved to possess the following analytical solutions $[25,26]$ :

(i) The bright soliton [see Fig. 1(a)]:

$$
u_{b s}(x, t)=2 b i \operatorname{sech}\left[2 b\left(x+16 \delta b^{4} t\right)\right] e^{2 i b^{2} t},
$$

for purely imaginary eigenvalues, $\lambda=b i$, where $b$ determines the amplitude of the soliton. In this case, the soliton has nonzero velocity, $v=-16 \delta b^{4}$. Additionally, the phase term $e^{2 i b^{2} t}$ evolves along the $t$ axis and does not depend on $x$. When $\delta=0$, it degenerates to the soliton solution of classical NLS equation.

(ii) The Akhmediev breather [see Fig. 1(b)]:

$$
u_{a b}(x, t)=\left[\frac{k^{2} \cosh (d t)+2 i d \sinh (d t)}{2 \cosh (d t)-2 a \cos \left[k\left(x+V_{1} t\right)\right]}-1\right] e^{i t},
$$

where $\lambda=a i(0<a<1), k=2 \sqrt{1+\lambda^{2}}=2 \sqrt{1-a^{2}}$ is the frequency of modulation, $d=$ $\frac{k}{2} \sqrt{4-k^{2}}=2 a \sqrt{1-a^{2}}$ is the growth rate of periodic modulation, and $V_{1}=\delta\left(k^{4}-10 k^{2}+30\right)=$ $\delta\left[\left(4 a^{2}+1\right)^{2}+5\right]$ defines the skewing angle. The variety of $\delta$ does not change the profile of solution qualitatively apart from skewing and stretching of the localization.

(iii) The Kuznetsov-Ma (KM) breather [see Fig. 1(c)]:

$$
u_{k m}(x, t)=\left[\frac{-f^{2} \cos (g t)-2 i g \sin (g t)}{2 \cos (g t)-2 c \cosh \left[f\left(x+V_{2} t\right)\right]}-1\right] e^{i t}
$$

where $\lambda=c i(c>1), f=2 \sqrt{c^{2}-1}, g=c f=2 c \sqrt{c^{2}-1}$, and $V_{2}=\delta\left(f^{4}+10 f^{2}+30\right)=$ $\delta\left[\left(4 c^{2}+1\right)^{2}+5\right]$.

(iv) The Rouge Wave (RW) [Localized in space and time, see Fig. 1(d)]:

$$
u_{r w}(x, t)=\left[\frac{4(1+2 i t)}{4(x+30 \delta t)^{2}+4 t^{2}+1}-1\right] e^{i t},
$$

which can be obtained from the limit $a \rightarrow 1$ in the AB (6) or $c \rightarrow 1$ in the KM breather (7), and it is a regular rational solution with one maximal point and two minimal points.

(a)

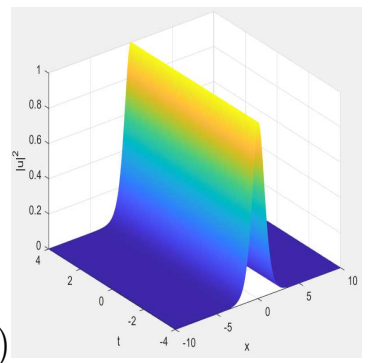

(b)

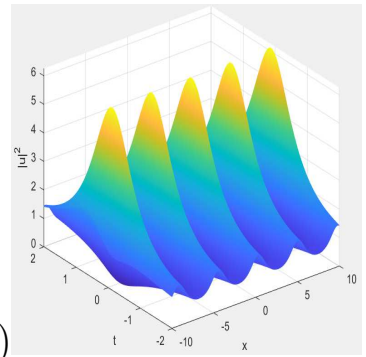

(c)

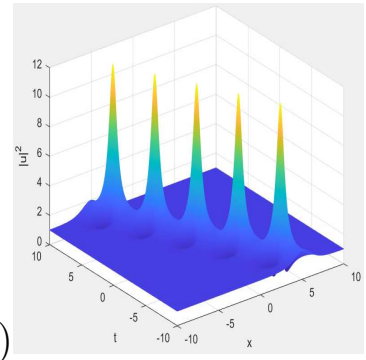

(d)

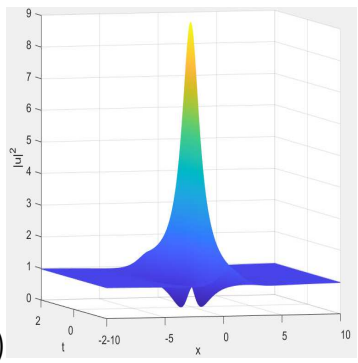

Figure 1 Analytical solutions of Eq. (1) with $\delta=0.01$. (a) The bright soliton with $b=0.5$; (b) the AB with $a=0.75$; (c) the KM breather with $c=1.2$; (d) the rogue wave. 


\subsection{Numerical computation of spectral portraits of the spatial Lax pair with distinct potentials}

As far as we know, there is no research on the spectral signatures of spatial Lax pair (2a) with distinct potentials of Eq. (1). Usually, it is difficult to analytically find the spectral portrait for a general potential $u(x, t)$. Therefore, some numerical methods have been proposed to study the IST spectra [27, 28]. Eq. (2a) can be rewritten as a standard linear eigenvalue problem [28],

$$
M \Phi=\lambda \Phi, \quad M=\left(\begin{array}{cc}
-i \partial x & -u^{*} \\
u & i \partial x
\end{array}\right),
$$

which can be subsequently solved by using the Fourier collocation method [29]. The $x$ axis is truncated into a finite interval $[-L / 2, L / 2]$, where $L$ is the length of the interval. Then, for a fixed $t=t_{0}$, the eigenvector $\Phi=\left(\Phi_{1}(x), \Phi_{2}(x)\right)^{T}$ as well as the potential $u(x, t)$ are expanded into Fourier series with $2 N+1$ modes,

$$
\Phi_{j}(x)=\sum_{n=-N}^{N} a_{j, n} e^{i n k_{0} x}, \quad j=1,2 ; \quad u(x, t)=\sum_{n=-N}^{N} c_{n} e^{i n k_{0} x},
$$

where $k_{0}=\frac{2 \pi}{L}$ and $t=t_{0}$ are given. Substituting these expansions into Eq. (9) and equating the coefficients of the same Fourier mode, one can obtain the eigenvalue spectrum.

(a1)

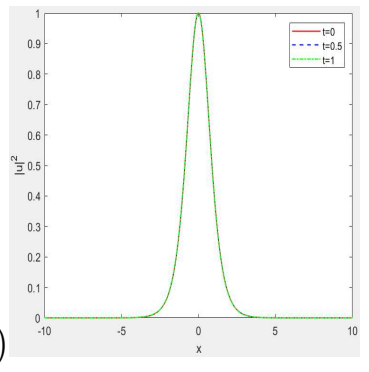

(a2)

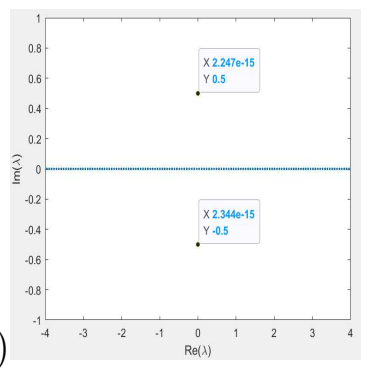

(b1)
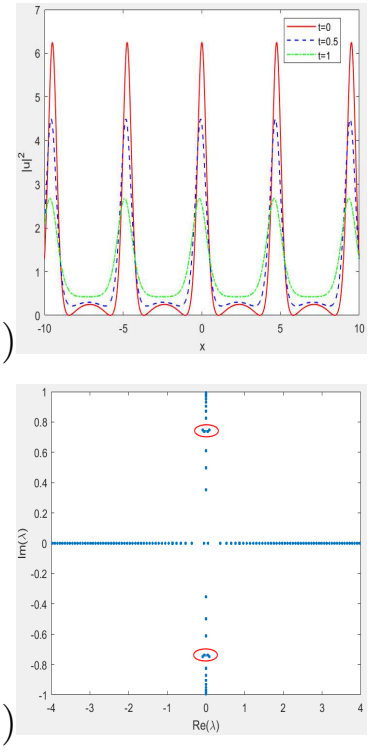

(c1)

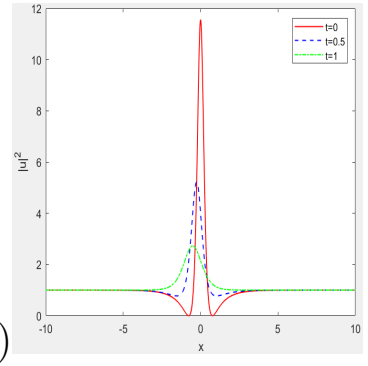

(d1)

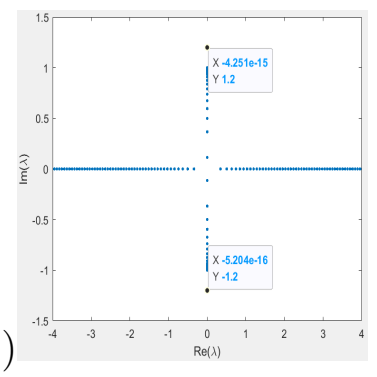

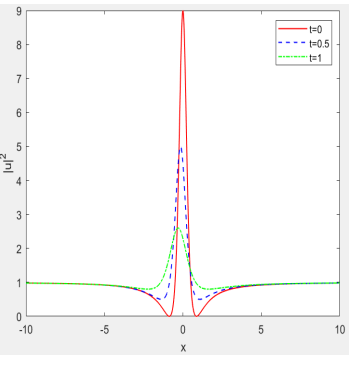

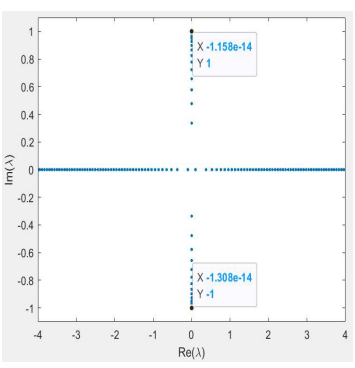

Figure 2 The numerical IST results. (a1)-(d1) Profiles of $|u(x, t)|^{2}$ in Fig. 1 at $t=0,0.5,1$; (a2)-(d2) the corresponding spectral portraits. A numerical interval of length $L=100$ discretized by 1000 points has been used to compute the eigenvalue spectra.

Fig. 2 shows the eigenvalue spectra of Eq. (9) with distinct potentials $u(x, t)$ (BS, AB, KM, RW). It can be seen that the BS (5) with $b=0.5$ has discrete eigenvalues of $\pm 0.5 i$, while the eigenvalue spectra of the other three potentials are all over the $(-1,1)$ interval of the imaginary axis. In addition, the $\mathrm{AB}(6)$ with $a=0.75$ has discrete eigenvalues around $\pm 0.75 i$, the KM (7) with $c=1.2$ has discrete eigenvalues of $\pm 1.2 i$, and the RW (8) has discrete eigenvalues of $\pm i$, 
respectively. Furthermore, it is worth noting that in our numerical results, although the potential $u(x, t)$ varies with the change of time $t$, the corresponding eigenvalue spectrum is always invariant.

\subsection{Spectral portraits of periodized structures}

In order to overcome the fact that a satisfactory numerical IST analysis of RWs cannot be generally achieved in a local way from a single isolated object, we usually need to use a local periodization procedure [18]. In this way, the spectral portraits can still be accurately determined from the numerical IST analysis of the periodized structures obtained from proper periodization of the isolated object.

Similar to $[19,23]$, we consider spectral portraits of periodized wavetrains in the Eq. (1). We extend the central part of the analytical solutions periodically at $t=0$, including the local peaks and the surroundings. In this way, the nonlinear interaction between the central peak and the surrounding structure can be effectively guaranteed, and the effective intensity of the interaction is determined by the spatial period $T$ that is used to produce the periodized wavetrains.

(a1)

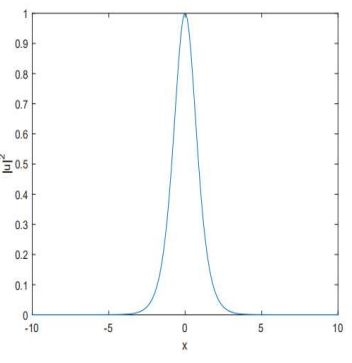

(b1)

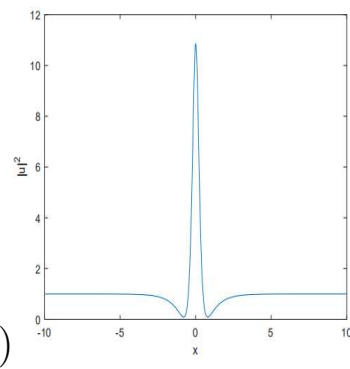

(a2)

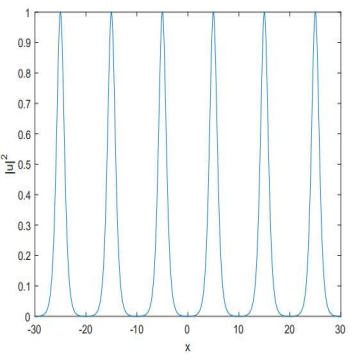

(a3)

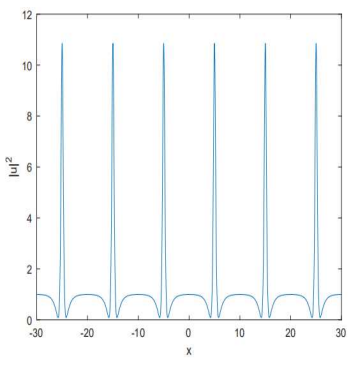

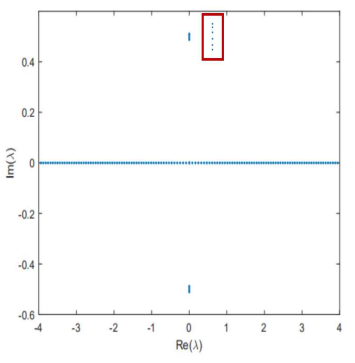

(b3)

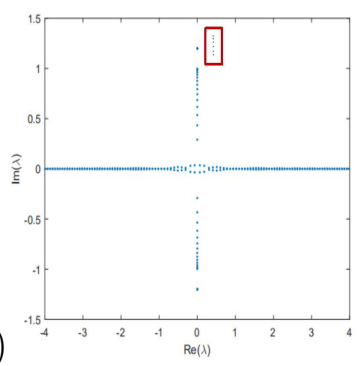

Figure 3 The numerical results of periodized structures. (a1), (b1) Profiles of $|u(x, t)|^{2}$ in Figs. 1(a) and 1(c) at $t=0$; (a2), (b2) the corresponding periodized wavetrains $(T=6)$; (a3), (b3) the spectral portraits of the periodized wavetrains showing that the periodization procedures produce same eigenvalue bands.

From Fig. 3, we can find that after the periodized extension of the central parts of the BS and KM breather solutions, although their potentials become more complex, the corresponding eigenvalue spectra do not change significantly, except that small bands are now found in Figs. 3(a3) and 3(b3) instead of single points. The discrete eigenvalues with a maximal norm in Figs. 2 (a2) and $2(\mathrm{c} 2)$ are $\pm 0.5 i$ and $\pm 1.2 i$, respectively, while the discrete eigenvalues are $\pm 0.4871 i$, $\pm 0.4916 i, \pm 0.4974 i, \pm 0.5034 i, \pm 0.5083 i, \pm 0.5116 i$ in Fig. $3(\mathrm{a} 3)$, and $\pm 1.197 i, \pm 1.198 i, \pm 1.199 i$, $\pm 1.2 i, \pm 1.2 i, \pm 1.201 i$ in Fig. 3(b3), respectively.

\section{Numerical simulations via symmetrical split-step Fourier method: the formation of rouge waves}

In this section, we simulate the evolution of Eq. (1) from distinct initial conditions by using the symmetrical split-step Fourier method [28]. We take $\delta=10^{-6}$ in the following numerical simulations, because Eq. (1) is very sensitive to the coefficient of the quintic terms. 


\subsection{The symmetrical split-step Fourier method}

The symmetrical split-step Fourier method simulates the interaction between the dispersion term and the nonlinear term by assuming that they work independently. Therefore, Eq. (1) can be rewritten as follows:

$$
\begin{aligned}
& \frac{\partial u}{\partial t}=M(u)+N(u), \\
& M(u)=\frac{i}{2} u_{x x}+\delta u_{x x x x x}, \\
& N(u)=i|u|^{2} u+10 \delta\left(|u|^{2} u_{x x x}+\left(\left|u_{x}\right|^{2} u\right)_{x}+2 u^{*} u_{x} u_{x x}+3|u|^{4} u_{x}\right),
\end{aligned}
$$

where $M$ and $N$ represent dispersion and nonlinear operators, respectively.

The idea of symmetrical split-step Fourier method is shown in Fig. 4. The propagation process from $t$ to $t+h$ is divided into three parts, in which the dispersion and nonlinear effects are assumed to work independently. First, the envelope $u(x, t)$ propagates half a step $\frac{h}{2}$, in which only the dispersion effectis considered, i.e., $N(u)=0$, and it can be transformed into ordinary differential equation to solve by Fourier transform; then, in the plane of $t+\frac{h}{2}$, only the nonlinear effect works, i.e., $M(u)=0$, and it can be solved by combining Fourier transform and four-order Runge-Kutta method; next, envelope propagates the remaining half step like the first part. In this way, we can obtain $u(x, t+h)$ representing the envelope after the evolution of one step.

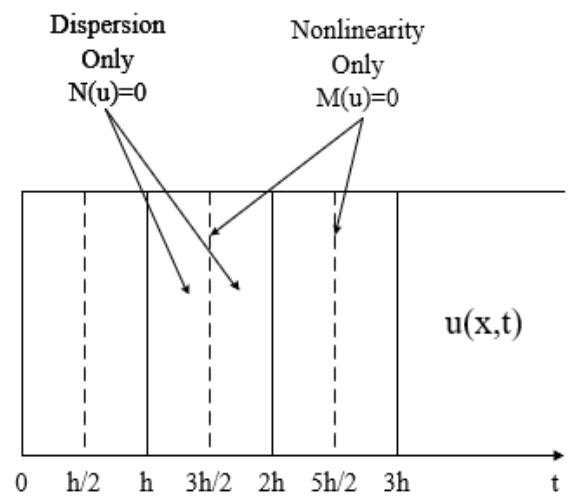

Figure 4 Numerical simulation process via symmetrical split-step Fourier method.

\subsection{Dam-break problem}

In this section, we first consider the dam-break problem $[23,30]$ as the initial condition,

$$
u(x, t=0)= \begin{cases}1, & -20<x<20 \\ 0, & 20 \leq|x| \leq 200\end{cases}
$$

to numerically simulate the wave propagation in the Eq. (1). The dam-break problem describes the evolution and interaction of two counter-propagating nonlinear wave trains generated in the quintic NLS box problem. We show that the interaction dynamics results in the emergence of modulated large-amplitude quasi-periodic breather lattices whose amplitude profiles are closely approximated by the Akhmediev breathers (AB) within certain space-time domain. [see Fig. 5]. 
(a)

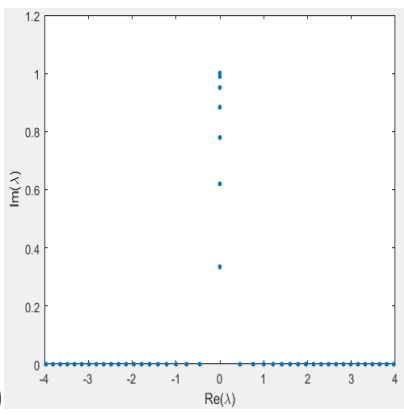

(b)

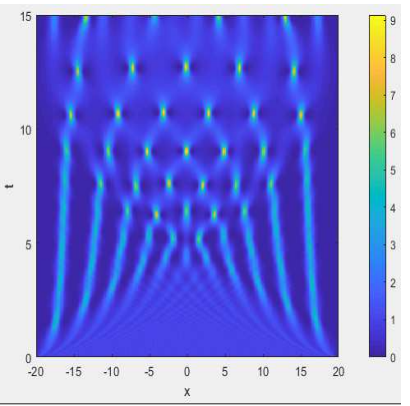

(c)

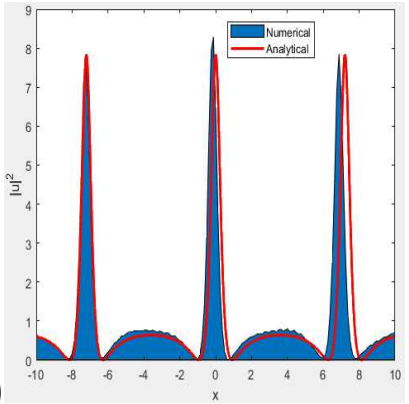

Figure 5 Numerical results of the dam-break problem. (a) The eigenvalue spectrum of the initial condition (12); (b) space-time diagram showing the evolution of $|u(x, t)|^{2}$ while starting from the initial condition (12) of Eq. (1) with $\delta=10^{-6}$. (c) the profile of the localized structure at $t=12.74$, compared with the analytical Akhmediev breather [Eq. (6)].

(a1)

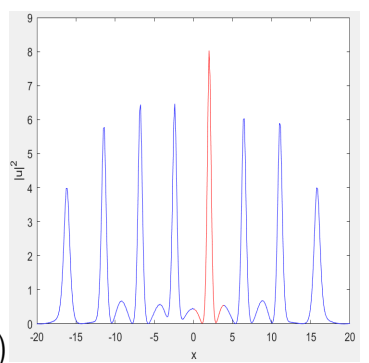

(b1)
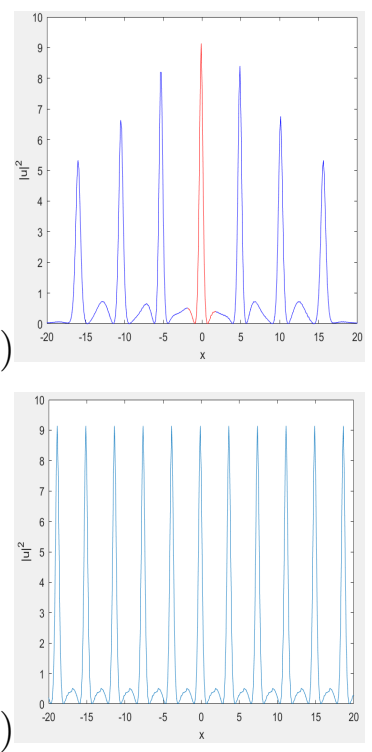

(b2)

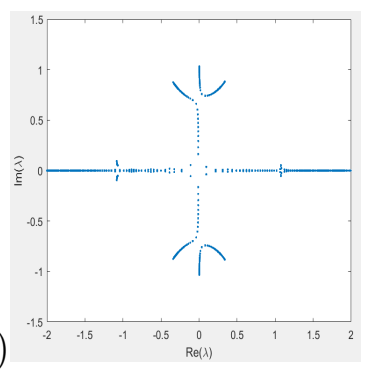

(b3)

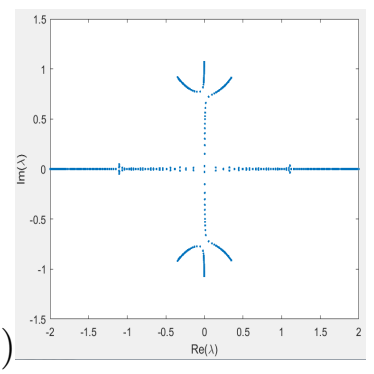

$(\mathrm{c} 1)$

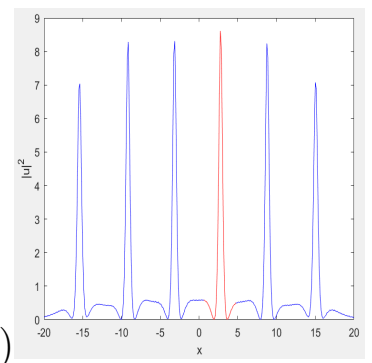

$(\mathrm{d} 1)$

(c2)

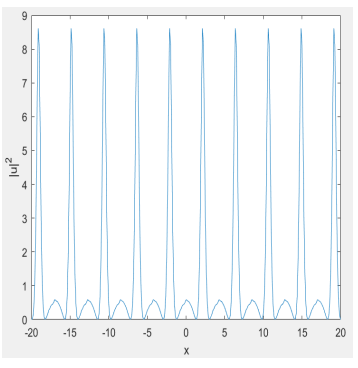

$(\mathrm{d} 2)$
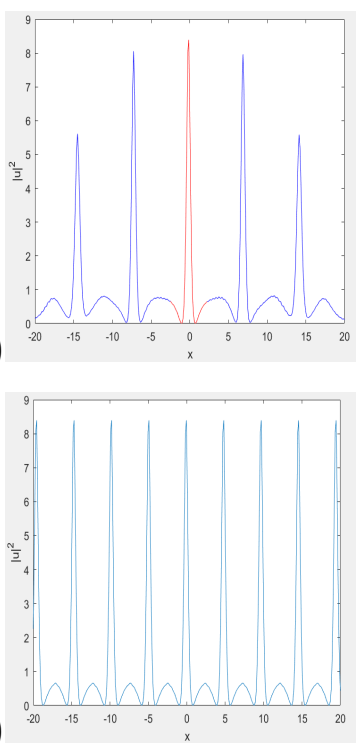

(c3)

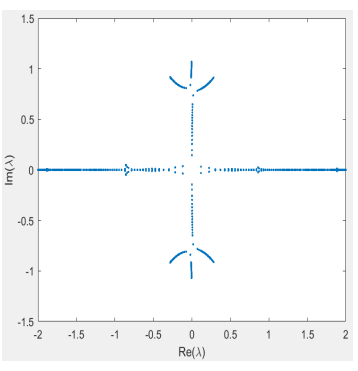

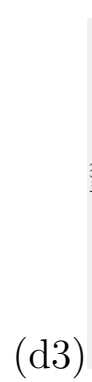

Figure 6 (a1)-(d1) The intensity $|u(x, t)|^{2}$ at $t=7.51,9.04,10.70,12.74$, respectively, and the parts of the profles that are highlighted in red represent the elementary patterns that are used to produce periodized wavetrains; (a2)-(d2) the corresponding periodized wavetrains; (a3)-(d3) the corresponding spectral portraits by numerical IST analysis of periodized wavetrains including 100 periods. 
Then, we take the localized structures observed at $t=7.51,9.04,10.70$ and 12.74 as examples, and extend the central part of them periodically to study their eigenvalue spectra by the Fourier oscillatory method and numerical IST analysis. Note that the criterion for choosing the period $T$ is that any sufficiently small change in $T$ can only results in enough small quantitative change in the spectrum. From Figs. 6(a3)-(d3), it can be found that these spectra are obviously different from Fig. 2(b2), which suggests that they are not identical to the analytical solutions. In fact, from the viewpoint of finite-gap theory, all the IST spectra possess 3 main spectral bands, which indicates that they are non-degenerate genus 2 solutions, while the AB, KM and RW are all degenerate genus 2 solutions [18].

\subsection{Noise-driven modulation instability and the formation of rogue waves}

For the nonlinear wave equations, the modulation instability (MI) may be regarded as a necessary condition for generating the extreme waves. In this section, we consider the plane wave plus a random noise as the initial condition,

$$
u(x, 0)=1+\epsilon \eta, \quad-20<x<20
$$

where $\eta$ is a random noise uniformly distributed in the $(-1,1)$ interval and $\epsilon$ is the intensity coefficient of the noise, to numerically simulate the wave evolution in the Eq. (1). We observe that compared with Fig. 5, the noise-driven MI results in a series of richer high-peak structures with random intensity in chaotic field, whose amplitude profiles are closely approximated by the $\mathrm{AB}, \mathrm{KM}$ and RWs [see Fig. 7].

(a)

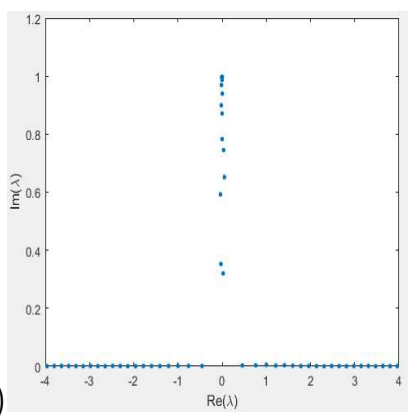

(b)

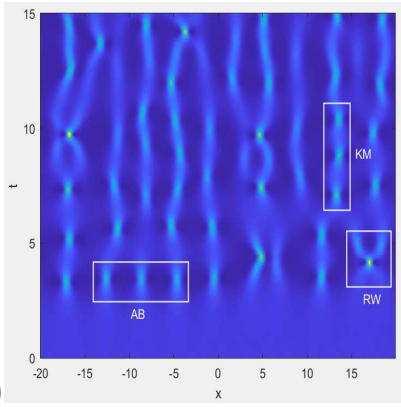

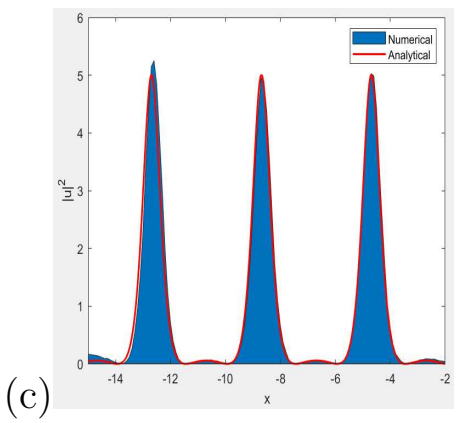

(d)

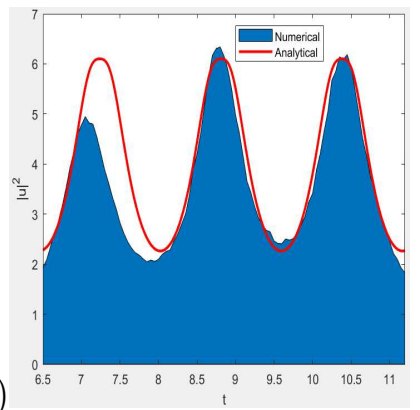

(e)

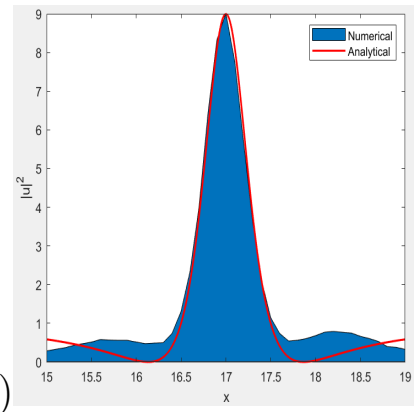

Figure 7 Numerical simulation driven by random noise. (a) The eigenvalue spectrum of the initial condition (13); (b) space-time diagram showing the evolution of $|u(x, t)|^{2}$ while starting from the initial condition (13) of Eq. (1) with $\delta=10^{-6}, \epsilon=0.02$; (c-e) localized structures extracted from the chaotic field in (a), compared with the analytical AB, KM and RW [Eqs. (6)-(8)].

In the evolution process, a series of irregular localized peak structures appear around $t=3$. Then, more complex periodic growth and decay behaviors emerge along the $t$ axis. Notice that 
the average spatial period here is around $\Delta x=\sqrt{2} \pi$, which corresponds to the reciprocal of the maximum MI gain frequency. Moreover, we compare the profiles of some high intensity peak structures with the corresponding analytical solutions in Eqs. (6)-(8), and find that they agree very well. Although it is impossible to observe the ideal analytic structures under the given random initial conditions, it is still worth noting how the analytic solutions can be closely mapped to the structures generated by the noise.

\subsection{Complex random initial condition and the formation of integrable turbulence fields}

However, the random noise $\eta$ in Eq. (13) is not related to the $x$. In order to make the curve smoother, we take the one-dimensional random rough surface as the initial condition,

$$
u(x, 0)=1+f(x), \quad f\left(x_{n}\right)=\frac{1}{L} \sum_{j=-N / 2+1}^{N / 2} F\left(k_{j}\right) e^{i k_{j} x_{n}}
$$

where $x \in[-L / 2, L / 2], L=100$, with a uniform grid of $N=1000$ nodes, $k_{j}=2 \pi j / L(j=$ $-N / 2+1, \cdots, N / 2), \Delta k=2 \pi / L, F\left(k_{j}\right)$ is defined as the Fourier transform of $f\left(x_{n}\right)$ as follows:

$$
F\left(k_{j}\right)=\frac{2 \pi}{\sqrt{2 \Delta k}} \sqrt{S\left(k_{j}\right)} \cdot \begin{cases}N(0,1)+i N(0,1), & j=-N / 2+1, \cdots, N / 2-1 \\ N(0,1), & j=0, N / 2\end{cases}
$$

where $S\left(k_{j}\right)$ is the power spectrum function of random rough surface obeying Gaussian distribution, and its expression is

$$
S\left(k_{j}\right)=\frac{\mu^{2} l}{2 \sqrt{\pi}} e^{-\frac{k_{j}^{2} l^{2}}{4}}
$$

where $\mu$ and $l$ represent standard deviation and correlation length, respectively.

In this way, we construct a complex random function $f(x)$, whose real and imaginary parts are independent random variables, each obeying the Gaussian distribution with standard deviation $\mu$ and correlation length $l$. The use of two variable parameters enables us to reveal more complex integrable chaotic dynamics than ever before.

(a)

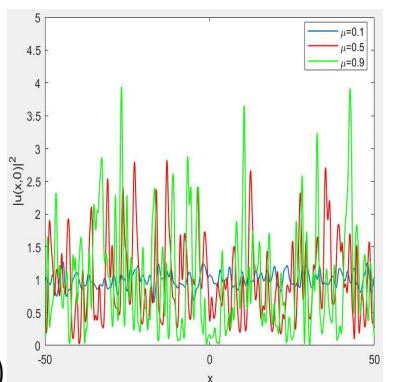

Figure 8 Initial field intensity $|u(x, 0)|^{2}$ for (a) fixed $l=0.76$ and three values of $\mu=0.1$ (the blue line), 0.5 (the red line), 0.9 (the green line); (b) fixed $\mu=0.5$ and three values of $l=0.19$ (the blue line), 0.76 (the red line), 1.9 (the green line). The value of the mean field intensity is taken always equal to 1 .

Fig. 8 shows the effect of these two variable parameters on the initial conditions. The random deviations from the $\mathrm{cw}$ with amplitude 1 increase with the increase of standard deviation $\mu$ [see Fig. $8(\mathrm{a})$ ]. However, when the correlation length $l$ increases, the deviations don't change significantly, but the period of the random waves becomes larger [see Fig. 8(b)]. 
Fig. 9 exhibits the numerical results for the initial conditions in Fig. 8(a). We numerically solve the eigenvalue problem Eq. (9) using these initial conditions and the sets of complex eigenvalues $\lambda$ are shown in Figs. 9(a1)-(c1). We can see that when $\mu=0.1$, eigenvalues are almost located on the imaginary axis, which correspond to the excitation of ABs. With the increase of the value $\mu$, the eigenvalues gradually deviate from the coordinate axis and turn to solitons, meaning that the chaotic pattern gradually changes from the breather turbulence to the soliton turbulence, which is consistent with the actual simulation results in Figs. 9(a2)-(c2). Figs. 9(a3)-(c3) dispaly part of the three-dimensional diagrams in the evolution process, which clearly illustrate the collision process of breathers and solitons. It should be emphasized that the superposition of the surrounding small radiation waves leads to the random irregular variation of the amplitudes in the collision process.

(a1)

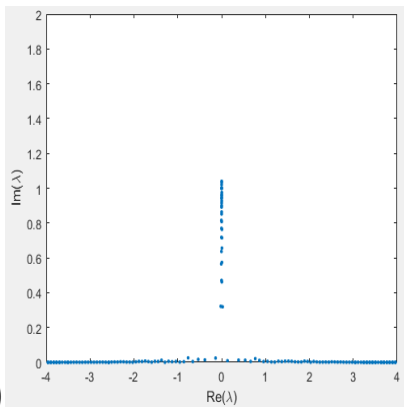

(b1)

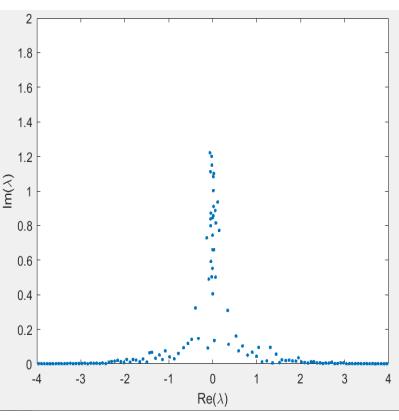

(c1)

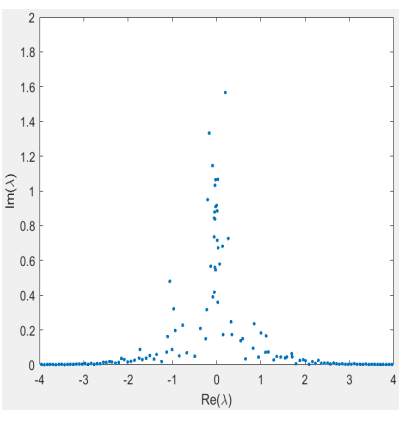

(a2)

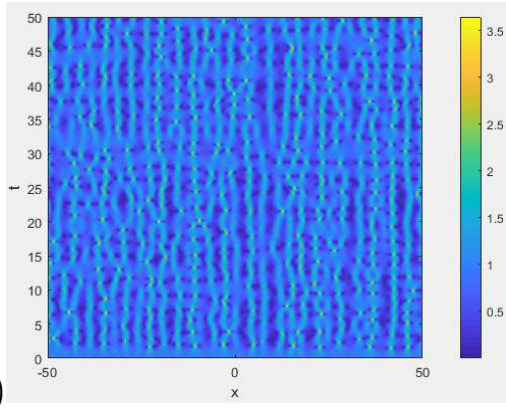

(b2)

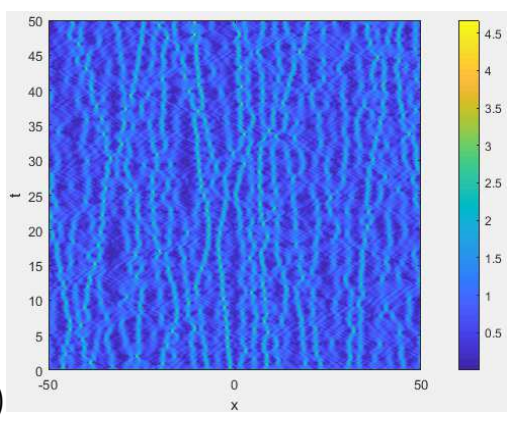

(c2)

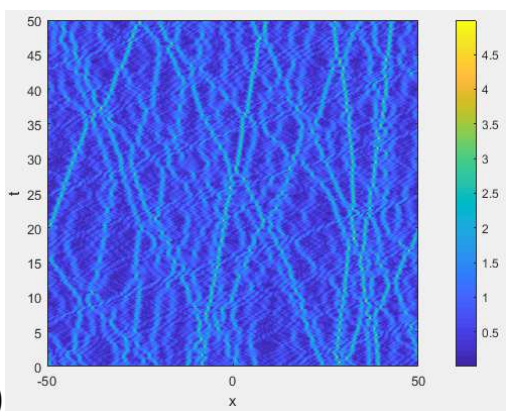

(a3)

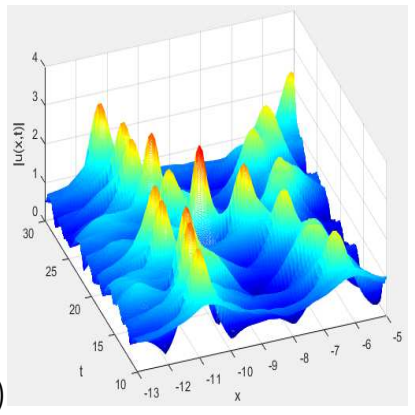

(b3)

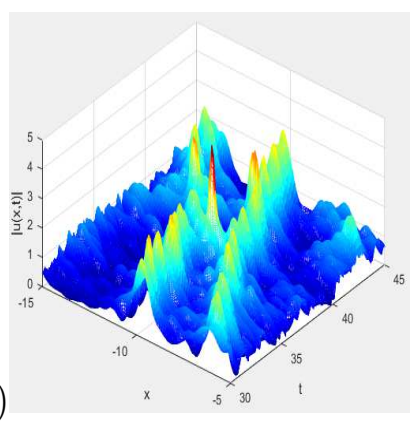

(c3)

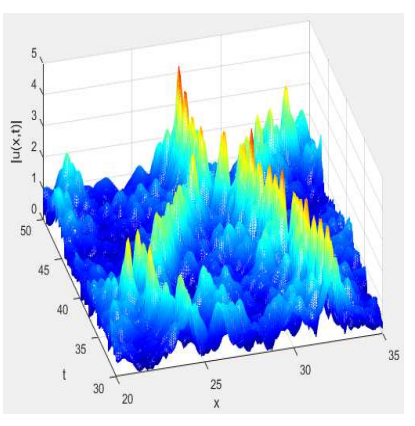

Figure 9 Numerical results for fixed $l=0.76$ and three values of $\mu=0.1,0.5,0.9$. (a1)-(c1) The eigenvalue spectra of the initial conditions; (a2)-(c2) the space-time diagrams showing the evolution of $|u(x, t)|^{2}$; (a3)-(c3) Parts of the chaotic patterns of (a2)-(c2) shown as 3D plots.

Another factor that plays a significant role in this chaotic evolution is the correlation length $l$ of the initial random field. Similarly, we exhibit the numerical results in Fig. 10 for the initial conditions in Fig. 8(b). Compared with Figs. 9(a1)-(c1), the eigenvalues in Figs. 10(a1)-(c1) still remain near the coordinate axis with the increase of $l$, but the range on the imaginary axis gradually expands, which means that the amplitudes of solitons in the initial field gradually increases. 
(a1)

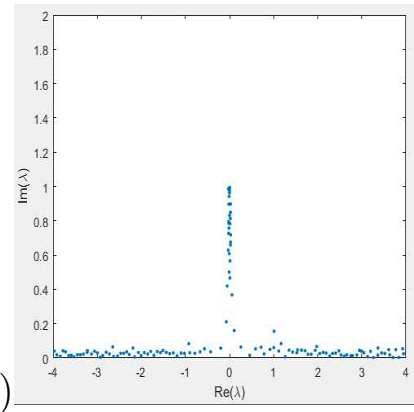

(b1)

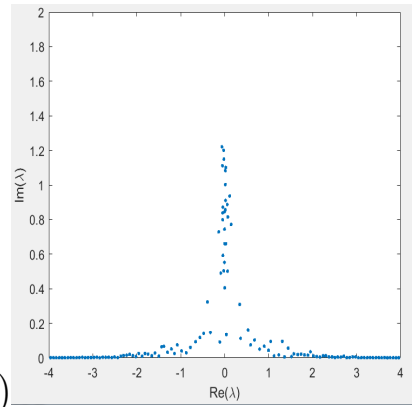

(c1)

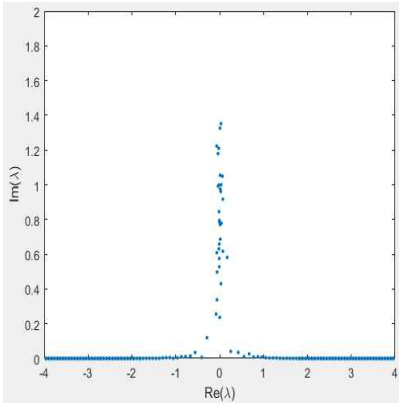

(a2)

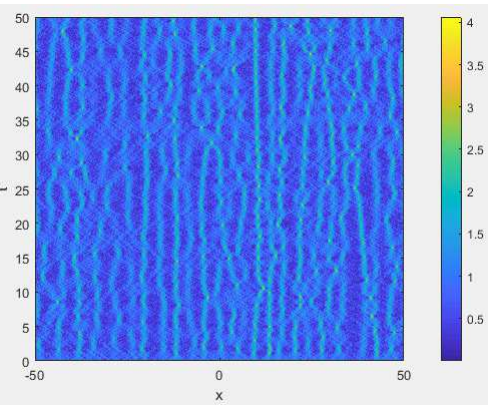

(b2)

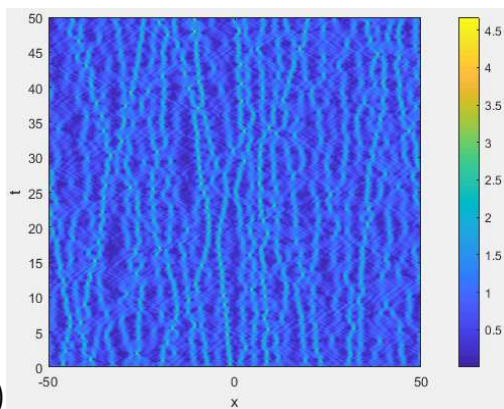

$(\mathrm{c} 2)$

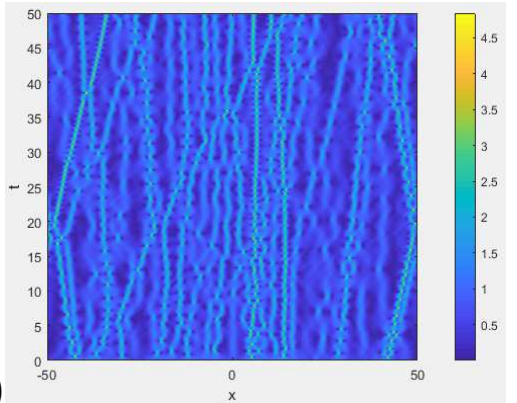

(a3)

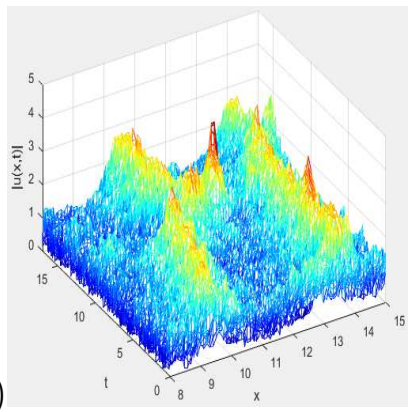

(b3)

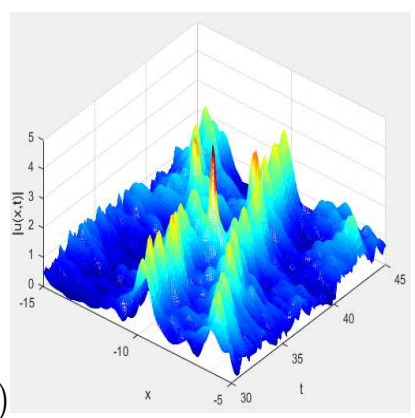

(c3)

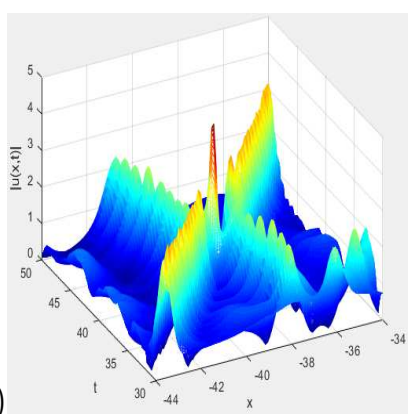

Figure 10 Numerical results for fixed $\mu=0.5$ and three values of $l=0.19,0.76,1.9$. (a1)-(c1) The eigenvalue spectra of the initial conditions; (a2)-(c2) the space-time diagrams showing the evolution of $|u(x, t)|^{2}$; (a3)-(c3) Parts of the chaotic patterns of (a2)-(c2) shown as 3D plots.

(a)

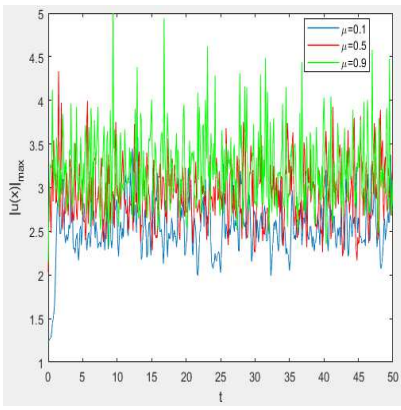

(b)

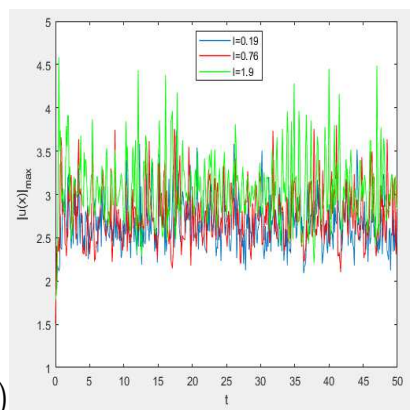

Figure 11 Evolution of the maximum of the field amplitudes versus $t$ for (a) fixed $l=0.76$ and three values of $\mu=0.1$ (the blue line), 0.5 (the red line), 0.9 (the green line); (b) fixed $\mu=0.5$ and three values of $l=0.19$ (the blue line), 0.76 (the red line), 1.9 (the green line). 
Fig. 11 illustrates the results of the simulations with the above initial conditions. It shows the peak amplitude (absolute maximum of $|u|$ over the whole $x$ interval of simulation) as a function of $t$. As expected from the theory of MI, the maximum increases exponentially at the initial stages of evolution, and then gradually enters into chaotic pattern. Moreover, with the increase of value $\mu$ or $l$, the convergence to the chaotic state is quicker and the amplitudes of RWs in the chaotic field are higher.

\section{Conclusions}

In this paper, we focus on the quintic nonlinear Schrödinger (NLS) equation. Firstly, we numerically study the spectral signatures of the spatial Lax pair with distinct analytical solutions [i.e., solitons, Akhmediev (AB), Kuznetsov-Ma (KM) breathers, and rogue waves (RWs)] of the quintic NLS equation through the Fourier oscillatory method. Secondly, we discuss the formation of rogue waves in the quintic NLS equation. Distinct non-zero initial conditions are used to analyze the generation mechanism of rogue waves: (i) the one case is based on a decaying initial condition (dam-break problem). We observe the modulated breather lattices approximated by the AB, and further compare their spectral signatures using periodization procedure. (ii) in another case, we use plane wave plus a random noise as the initial condition such that the modulation instability (MI) seeded from the noise results in a series of richer high-peak structures of random intensity, and they agree well with the analytical solutions. (iii) in the last case, we take the one-dimensional random rough surface as the initial condition and analyze the formation of rogue waves. We find that two parameters of the initial random functions are essential to fix the number of rogue waves in the resulting chaotic wave field: $\mu$ influences the relative number of breathers and solitons in the resulting chaotic wave field while $l$ mainly influences the amplitudes of the solitons. They all lead to an increase in the amplitude of rogue waves. The results obtained in this paper will be useful to understand the formation of rogue waves in the quintic NLS equation and other related models, which may provide ideas for the controllable excitation of rogue waves in nonlinear fields such as optical fibers.

\section{Declarations}

\section{Funding}

This research is supported by the National Natural Science Foundation of China (No.11972291), Natural Science Basic Research Program of Shaanxi (No.2020JM-115) and Aeronautical Science Foundation of China (No.201941053004).

\section{Data availability statement}

The datasets generated during and/or analysed during the current study are available from the corresponding author on reasonable request.

\section{References}

[1] Ruan H Y and Chen Y X. The study of exact solutions to the nonlinear Schrödinger equations in optical fiber. Journal of the Physical Society of Japan. 2007,72(6):1350.

[2] Wang Y Y, Li J T, Dai C Q, et al. Solitary waves and rogue waves in a plasma with nonthermal electrons featuring Tsallis distribution. Physics Letters A. 2013,377(34-36):2097-2104.

[3] Ma W X. Interaction solutions to Hirota-Satsuma-Ito equation in (2+1)-dimensions. Frontiers of Mathematics In China. 2019,14(3):619-629. 
[4] Zhang L J, Han M A, Khalique C M, et al. A new type of solitary wave solution of the $\mathrm{mKdV}$ equation under singular perturbations. International Journal of Bifurcation and Chaos. 2020,30(11):2050162.

[5] Khalique C M and Simbanefayi I. Conservation laws, classical symmetries and exact solutions of a $(1+1)$-dimensional fifth-order integrable equation. International Journal of Geometric Methods in Modern Physics. 2021,18(09):2150137.

[6] Lou Y, Zhang Y, Ye R S, et al. Solitons and dynamics for the integrable nonlocal pairtransition-coupled nonlinear Schrödinger equation. Applied Mathematics and Computation. 2021,409:126417.

[7] Tang Y N, He C H and Zhou M L. Darboux transformation of a new generalized nonlinear Schrödinger equation: soliton solutions, breather solutions, and rogue wave solutions. Nonlinear Dynamics. 2018,92(4):2023-2036.

[8] He C H, Tang Y N, Ma W X, et al. Interaction phenomena between a lump and other multi-solitons for the (2+1)-dimensional BLMP and Ito equations. Nonlinear Dynamics. 2019,95(1):29-42.

[9] Zhou J L, Tang Y N and Zhang L P. Modulation instability and rogue wave spectrum for the generalized nonlinear Schrödinger equation. Physica Scripta. 2020,95(11):115205.

[10] Tang Y N and Zhou J L. Mixed interaction solutions for the coupled nonlinear Schrödinger equations. Modern Physics Letters B. 2021,35(10):2150004.

[11] Akhmediev N, Soto-Crespo J M and Ankiewicz A. Extreme waves that appear from nowhere: On the nature of rogue waves. Physics Letters A. 2009,373(25):2137-2145.

[12] Solli D R, Ropers C, Koonath P, et al. Optical rogue waves. Nature. 2007,450(7172):10541057.

[13] Kharif C, Pelinovsky E and Slunyaev A. Rogue Waves in the Ocean. 2009.

[14] Cao D B, Pan L X, Sun Y B, et al. Families of exact solutions for two-component Bose-Einstein condensates. Advanced Materials Research. 2010,152-153:1309-1312.

[15] Yan Z Y. Financial rogue waves. Communications in Theoretical Physics. 2010,11:947-949.

[16] Onorato M, Residori S, Bortolozzo U, et al. Rogue waves and their generating mechanisms in different physical contexts. Physics Reports. 2013,528(2):47-89.

[17] Dudley J M, Dias F, Erkintalo M, et al. Instabilities, breathers and rogue waves in optics. Nature Photonics. 2014, 8(10):755-764.

[18] Randoux S, Suret P and El G. Inverse scattering transform analysis of rogue waves using local periodization procedure. Scientific Report. 2016,6(474):29238.

[19] Soto-Crespo J M, Devine N and Akhmediev N. Integrable turbulence and rogue waves: breathers or solitons?. Physical Review Letters. 2016,116(10):103901. 
[20] Akhmediev N, Soto-Crespo J M and Devine N. Breather turbulence versus soliton turbulence: rogue waves, probability density functions, and spectral features. Phys.rev.e. 2016,94(2):022212.

[21] Onorato M, Osborne A R and Serio M. Modulational instability in crossing sea states: a possible mechanism for the formation of freak Waves. Physical Review Letters. 2006,96(1):014503.

[22] Biondini G and Fagerstrom E. The integrable nature of modulational instability. SIAM Journal on Applied Mathematics. 2015,75(1):136-163.

[23] Wang L, Yan Z Y and Guo B L. Numerical analysis of the Hirota equation: Modulational instability, breathers, rogue waves, and interactions. Chaos. 2020,30(1):013114.

[24] Yang Y, Yan Z Y and Malomed B A. Rogue waves, rational solitons, and modulational instability in an integrable fifth-order nonlinear Schrödinger equation. Chaos: An Interdisciplinary Journal of Nonlinea. 2015,88(6):193.

[25] Chowdury A, Kedziora D J, Ankiewicz A, et al. Soliton solutions of an integrable nonlinear Schrödinger equation with quintic terms. Phys Rev E Stat Nonlin Soft Matter Phys. 2014,90(3):032922.

[26] Kedziora D J, Akhmediev N, Chowdury A, et al. Breather solutions of the integrable quintic nonlinear Schrödinger equation and their interactions. Physical review, E. Statistical, nonlinear, and soft matter physics. 2015,91(2):022919.

[27] Boffetta G and Osborne A R. Computation of the direct scattering transform for the nonlinear Schrödinger equation. Journal of Computational Physics. 1992,102(2):252-264.

[28] Yang J K. Nonlinear waves in integrable and nonintegrable systems. Society for Industrial and Applied Mathematics. 2010.

[29] Boyd J P, Marilyn T and Eliot P. Chebyshev and Fourier Spectral Methods. Dover Publications. 2020.

[30] El G A, Khamis E G and Tovbis A. Dam break problem for the focusing nonlinear Schrödinger equation and the generation of rogue waves. Nonlinearity. 2016,29(9):2798-2836. 